\title{
DOSAGE PLASMATIQUE ET GLOBULAIRE DU MAGNESIUM DANS L'EXPLORATION DE LA RHINITE ALLERGIQUE
}

\author{
G. ONDZOTTO, M. DIATEWA *, T. FOUEMINA, J. KOUTIHOU**, F. BISSIKO \\ SERVICE ORL, CHU DE BRAZZAVILLE, CONGO \\ *LABORATOIRE DE BIOCHIMIE. FACULTÉ DES SCIENCES DE LA SANTÉ. UNIVERSITÉ MARIEN \\ NGOUABI. BRAZZAVILLE CONGO \\ **LABORATOIRE D’ANALYSES MÉDICALES «COGEMO », BRAZZAVILLE CONGO
}

\begin{abstract}
OBJECTIFS : La rhinite allergique représente un véritable problème de santé publique. Le but de cette étude est d'évaluer l'intérêt du dosage plasmatique et globulaire du magnésium dans le diagnostic de la rhinite allergique.

Matériel et méthodes : Etude analytique et prospective de 80 dossiers, sur une période de 4 ans et 5 mois (janvier 2004 à mai 2008), incluant les patients présentant une rhinite allergique, appariés à 80 témoins pour le sexe et l'âge. Les prélèvements sanguins ont été effectués dans tous les cas dans les mêmes conditions, le matin à jeun. Le dosage du magnésium a été réalisé grâce à un spectrophotomètre d'absorption atomique (SCREEN MASTER) selon la méthode colorimétrique. Les paramètres d'étude ont été les suivants : caractéristiques épidémiologiques et aspects cliniques de la rhinite allergique ; taux moyens de magnésium plasmatique et globulaire, les seuils pathologiques observés chez les témoins ; valeurs du magnésium plasmatique et globulaire observées chez les malades.

Résultats : Notre série comportait 43,75\% d'hommes et 56,25\% de femmes, âgés de 6 à 45 ans (moyenne d'âge : 27 ans). Les tranches de $16-35$ ans représentent $76,25 \%$ des cas. Les antécédents personnels $(20 \%)$ et familiaux $(60 \%)$ d'atopie étaient retrouvés. La durée d'évolution de la rhinite était de 1 à 20 ans (moyenne : 9 ans). La triade classique faite de rhinorrhée aqueuse (72\%), éternuements en salves $(60,8 \%)$ et d'obstruction nasale $(58,2 \%)$; ainsi que la pâleur pituitaire $(56,2 \%)$, le prurit $(50,25 \%)$ étaient les éléments cliniques dominants. La maladie évoluait sous la forme persistante dans la totalité des cas.

Les témoins présentaient : un taux moyen de magnésium plasmatique de $21,1 \mathrm{mg} / \mathrm{l} \pm 0,98$ et un seuil pathologique de $15,7 \mathrm{mg} / \mathrm{l}<21,1$ $\mathrm{mg} / \mathrm{l}<26,5 \mathrm{mg} / \mathrm{l}$; un taux moyen de magnésium globulaire de $57,05 \mathrm{mg} / \mathrm{l} \pm 2,42$ et un seuil pathologique de $44,79 \mathrm{mg} / \mathrm{l}<57,05 \mathrm{mg} / \mathrm{l}<$ $69,31 \mathrm{mg} / \mathrm{l}$. Les sujets malades avaient dans $50 \%$ des cas une magnésémie plasmatique abaissée ; $68,75 \%$ des cas une magnésémie globulaire abaissée ; $37,5 \%$ des cas une magnésémie plasmatique et globulaire abaissée $; 23,75 \%$ des cas une magnésémie globulaire pathologique ; $5 \%$ des cas une magnesémie plasmatique pathologique ; 3,75\% des cas une magnésémie plasmatique et globulaire pathologique.

Conclusion : L'hypomagnésémie concerne plus la fraction globulaire que plasmatique. Le dosage de la magnesémie est un élément d'orientation para-clinique dans le diagnostic de la rhinite allergique. La magnésothérapie présente un intérêt certain dans la prise en charge de cette pathologie.

Mots-clés : Magnésium, Globulaire, Plasmatique, Rhinite allergique.
\end{abstract}

Objectives: The allergic rhinitis represents a real public health problem. The goal of this survey is to value the interest of the dosage plasmatical and globular of magnesium in the diagnosis of the allergic rhinitis.

Materials and methods : Analytic and prospective survey of 80 files, on one period of 4 years and 5 months (from January 2004 to may 2008), including the patients presenting an allergic rhinitis, matched to 80 witnesses for the sex and age. The blood withdrawals have been done in any case in the same conditions, the morning on an empty stomach. The dosage of magnesium has been achieved thanks to an atomic absorption spectrophotometer (SCREEN MASTER) according to the method colorimetrical. The parameters of survey were the next one: epidemiological features and clinical aspects of the allergic rhinitis; middle rates of magnesium plasmatical and globular, the pathological doorsteps observed among the witness; values of magnesium plasmatical and globular lowered and pathological observed at the patients.

Results : Our set included $43.75 \%$ of men and $56.25 \%$ of women aged of 6-45 years (average of age: 27 years). The slices of $16-35$ years represent $76.25 \%$ of the cases. The personal antecedents $(20 \%)$ and familiar $(60 \%)$ atopy was recovered. The length of the rhinitis was of 1-20 years (average: 9 years). The classic triad made of aqueous rhinorrea (72\%), sneezes in salvos $(60.8 \%)$ and nasal obstruction $(58.2 \%)$; as well as the pituitary paleness $(56.2 \%)$, the pruritus $(50.25 \%)$ were the dominant clinical elements. The illness evolved under the obstinate shape in the totality of the cases.

The witnesses presented: a middle rate of magnesium plasmatical of $21.1 \mathrm{mg} / \mathrm{l} \pm 0.98$ and a pathological doorstep of $15.7 \mathrm{mg} / \mathrm{l}<21.1$ $\mathrm{mg} / \mathrm{l}<26.5 \mathrm{mg} /$; a middle rate of globular magnesium of $57.05 \mathrm{mg} / \mathrm{l} \pm 2.42$ and a pathological doorstep of $44.79 \mathrm{mg} / /<57.05 \mathrm{mg} / \mathrm{l}<69.31 \mathrm{mg} / \mathrm{l}$. The sick topics had in: $50 \%$ of the cases a lowered magnesium plasmatical; $68.75 \%$ of the cases lowered globular magnesium; $37.5 \%$ of the cases a magnesium plasmatical and globular lowered; $23.75 \%$ of the cases pathological globular magnesium; $5 \%$ of the cases a magnesium pathological plasmatic; $3.75 \%$ of the cases a magnesium plasmatical and globular pathological.

Conclusion: The decrease of magnesium in blood concerns the globular fraction more than plasmatic. The dosage of the magnesium is an element of decorate-clinic orientation in the diagnosis of the allergic rhinitis. The magnesotherapy presents a certain interest in the hold in charge of this pathology.

Key words: Magnesium, Globular, Plasmatic, Allergic Rhinitis. 


\section{INTRODUCTION}

La Rhinite allergique (RA) est l'inflammation allergique des voies aériennes supérieures. Elle représente un véritable problème de santé publique, affectant entre 10 et $25 \%$ de la population globale $(1,2)$.

Bien qu'il ne s'agisse pas d'une maladie grave, la RA peut néanmoins altérer la qualité de vie des sujets qui en souffrent, réduisant notamment la capacité d'apprentissage des enfants et des adolescents et les performances du travail des adultes. Les coûts directs et indirects qui lui sont attribués sont importants pour les pays en voie de développement $(3,4)$.

Dans le cadre de l'exploration de la RA, le bilan para clinique comprend, parmi les examens d'orientation, le dosage plasmatique et globulaire du magnésium (5). Ce dernier jouerait un rôle de modificateur du terrain vis-à-vis de l'allergie. II s'est agi dans ce travail, d'évaluer l'intérêt du dosage sanguin du magnésium dans le diagnostic de RA.

\section{MATÉRIEL ET MÉTHODES}

L'étude analytique et prospective sur une période de 4 ans et 5 mois (janvier 2004 à mai 2008), a été réalisée au Centre Hospitalier Universitaire de Brazzaville, à partir des dossiers des patients chez qui un dosage plasmatique et globulaire du magnésium a pu être effectué. Ce dosage a été demandé devant :

- Les symptômes évoquant classiquement la RA, associant la triade : obstruction nasale, éternuements en salves et rhinorrhée séreuse ;

- Les signes qui sont parfois au premier plan d'une RA : prurit nasal, palatin, auriculaire, oculaire avec larmoiement ; jetage postérieur ; céphalées ; troubles olfactifs ;

- Les éléments orientant vers une allergie :

. Âge : 3 à 35 ans (la RA débutant rarement avant l'âge de 3 ans et après l'âge de 35 ans, sauf circonstances particulières) ;

. Antécédents personnels ou familiaux d'atopie, eczéma, urticaire et asthme ;

- Caractères évolutifs des signes orientant vers l'allergie persistante ou intermittente.

- La confirmation d'une RA par des examens complémentaires biologiques (phadiotop, IgE spécifiques) ou par des tests cutanés (prick-test).

Notre échantillon était composé de 80 patients présentant une RA.

Pour les besoins de notre étude, 80 patients témoins ont été choisis dans la population de Brazzaville, appariés pour le sexe et l'âge avec la population malade. Ceux-ci ont été sélectionnés après un interrogatoire et un examen clinique minutieux. Ils n'avaient pas d'antécédents personnels ni familiaux d'atopie et d'allergie clinique décelable. Ils ne présentaient pas de rhinite inflammatoire, ni des manifestations d'inflammation des voies respiratoires basses. La réponse aux tests multi-allergiques de dépistage était négative.
Dans cette sélection, les patients exclus ont été ceux présentant une atteinte du système neuromusculaire, digestif et immunitaire.

Les prélèvements de sang ont été effectués chez les malades et les témoins dans les mêmes conditions, le matin à jeun. Le dosage du magnésium a été effectué dans le laboratoire COGEMO, grâce à un spectrophotomètre d'absorption anatomique (SCREEN MASTER), selon la méthode colorimétrique. L'intensité de la coloration, proportionnelle à la concentration en magnésium, permet un dosage photométrique à 500 millimicrons. Les valeurs de référence indiquées par ledit laboratoire étaient les suivantes :

- Magnésium plasmatique : 19-25 mg/l ;

- Magnésium globulaire : $\geq 50 \mathrm{mg} / \mathrm{l}$, pouvant atteindre

$70 \mathrm{mg} / \mathrm{l}$.

Les paramètres d'étude étaient :

- Population d'étude : caractéristiques épidémiologiques et cliniques de la RA;

- Les taux moyens de magnésium plasmatique et globulaire, les seuils pathologiques observés chez les témoins; -Les valeurs du magnésium plasmatique et globulaire abaissées et pathologiques observées chez les malades. Pour l'analyse et l'interprétation des résultats, les variables statistiques suivantes ont été étudiées :

- La moyenne et la variance ;

- Le taux moyen de la magnésemie estimé à :

$\delta$ : écart type ; 1,96 : écart réduit ; $n$ : nombre de sujets. - Le seuil pathologique (aux limites duquel une magnésémie doit être considérée comme pathologique) estimé à $\mathrm{X} \pm 1.96 \delta$.

La marge d'erreur était de 0,05.

\section{RÉSULTATS}

- Population d'étude : patients présentant une RA - Caractéristiques épidémiologiques

Notre étude comportait 80 cas de RA dont 35 hommes $(43,75 \%)$ et 45 femmes $(56,25 \%)$. L'âge était compris entre 6 ans et 45 ans avec une moyenne de 27 ans. Les tranches de 16 à 35 ans représentaient $76,25 \%$ des patients (tableau I).

\begin{tabular}{|c|c|c|c|c|}
\hline \multirow{2}{*}{ AGE (ans) } & \multicolumn{2}{|c|}{ SEXE } & \multirow{2}{*}{$\begin{array}{c}\text { Nombre } \\
\text { de cas }\end{array}$} & $\begin{array}{c}\text { Pourcentage } \\
\text { (\%) }\end{array}$ \\
\cline { 2 - 5 } & Homme & Femme & 9 & 11,25 \\
\hline $6-15$ & 5 & 4 & 20 & 25 \\
\hline $16-25$ & 8 & 12 & 41 & 51,25 \\
\hline $26-35$ & 17 & 24 & 10 & 12,5 \\
\hline $36-45$ & 5 & 5 & 80 & 100 \\
\hline Total & 35 & 45 & \multicolumn{2}{c}{} \\
\hline
\end{tabular}

Tableau I: Age et sexe 


\section{. Caractéristiques cliniques}

Les antécédents personnels et familiaux d'atopie étaient retrouvés respectivement dans $20 \%$ et $60 \%$ des cas.

La durée d'évolution de la RA était de 1 à 20 ans (moyenne : 9 ans).

Les éléments de l'examen clinique, retrouvés isolément ou associés ont été résumés dans le tableau II. Les symptômes prédominants étaient la rhinorrhée aqueuse (72\%), les éternuements en salve $(60,8 \%)$ et l'obstruction nasale $(58,2 \%)$. Les manifestations étaient persistantes et sans horaire précis, mais plus remarquables le matin dans tous les cas (tableau II).

\begin{tabular}{|c|c|c|}
\hline Elément clinique & $\begin{array}{c}\text { Nombre de cas (effectif } \\
\text { cumulé) }\end{array}$ & $\begin{array}{c}\text { Pourcentage } \\
\text { (\%) }\end{array}$ \\
\hline Rhinorrhée aqueuse & 58 & 72 \\
\hline Eternuements en salves & 49 & 60,8 \\
\hline Obstruction nasale & 47 & 58,2 \\
\hline Pituitaire pâle & 45 & 56,2 \\
\hline Prurit & 40 & 50,25 \\
\hline Pituitaire lilas ou violacée & 21 & 26,8 \\
\hline Céphalées & 16 & 20,35 \\
\hline Pituitaire inflammatoire & 12 & 15 \\
\hline Jetage postérieur & 8 & 10 \\
\hline Troubles de l'olfaction & 6 & 7,8 \\
\hline
\end{tabular}

Tableau II : Eléments de l'examen clinique

\section{. Etude de la magnésemie plasmatique et globulaire} Dans la population témoin, le taux moyen de magnésemie globulaire était de $57,05 \mathrm{mg} / \mathrm{l} \pm 2,42$ et celui de la magnésemie plasmatique de 21,1 mg/l $\pm 0,98$ (tableau III).

\begin{tabular}{|c|c|c|}
\hline Magnésémie & Taux moyen & Seuil pathologique \\
\hline Plasmatique & $21,1 \mathrm{mg} / \mathrm{l} \pm 0,98$ & $15,7 \mathrm{mg} / \mathrm{l}<21,1 \mathrm{mg} / \mathrm{l}<26,5 \mathrm{mg} / \mathrm{l}$ \\
\hline Globulaire & $57,05 \mathrm{mg} / \mathrm{l} \pm 2,42$ & $44,79 \mathrm{mg} / \mathrm{l}<57,05 \mathrm{mg} / 1<69,3 \mathrm{mg} / \mathrm{l}$ \\
\hline
\end{tabular}

Tableau III : Magnésemie plasmatique et globulaire des sujets témoin

Les résultats des différentes analyses faites chez les sujets ayant une RA ont été portés dans les tableaux IV et V. Dans $50 \%$ des cas, la magnésemie plasmatique était inférieure à 20,12 mg/l (tableau IV). La magnésemie globulaire était inférieure à $54,71 \mathrm{mg} / \mathrm{l}$ dans $68,7 \%$ des cas (tableau IV). La magnésemie globulaire était inférieure au seuil pathologique dans $23,75 \%$ des cas (tableau V). La magnésemie plasmatique et globulaire était abaissée dans 30 cas $(23,75 \%)$. Trois (3) cas $(3,75 \%)$ avaient à la fois une magnésemie plasmatique et globulaire pathologique. Dans tous les cas, la prédominance féminine était retrouvée.

\begin{tabular}{|c|c|c|}
\hline Sujet & $\begin{array}{c}\text { Magnésemie plasmatique } \\
(<\mathbf{2 0 , 1 2} \mathbf{~ m g / l )}\end{array}$ & $\begin{array}{c}\text { Magnésemie globulaire } \\
(<\mathbf{5 4 , 7 1} \mathbf{~ m g / l )}\end{array}$ \\
\hline Homme & $16(20 \%)$ & $25(31,25 \%)$ \\
\hline Femme & $24(30 \%)$ & $30(37,50 \%)$ \\
\hline Total & $40(50 \%)$ & $55(68,75 \%)$ \\
\hline
\end{tabular}

Tableau IV : Présentation des malades ayant une magnésemie en dessous de la normale

\begin{tabular}{|c|c|c|}
\hline Sujet & $\begin{array}{c}\text { Magnésemie plasmatique } \\
\text { inférieure au seuil } \\
\text { pathologique }\end{array}$ & $\begin{array}{c}\text { Magnésemie globulaire } \\
\text { inférieure au seuil } \\
\text { pathologique }\end{array}$ \\
\hline Homme & - & $9(11,25 \%)$ \\
\hline Femme & $4(5 \%)$ & $10(12,5)$ \\
\hline Total & $4(5 \%)$ & $19(23,75)$ \\
\hline
\end{tabular}

Tableau V : Présentation des sujets ayant une magnésemie pathologique

\section{DISCUSSION}

Caractéristiques épidémiologiques de la population malade et aspects cliniques de la RA :

Les RA représentent $30 \%$ des consultations ORL au Centre Hospitalier et Universitaire de Brazzaville (4). L'âge et le sexe ne semblent pas déterminer un rôle important dans la survenue des RA. Cependant, ils peuvent prédominer en fonction de l'allergie causale (5). Sa fréquence est importante chez l'adolescent et l'adulte jeune et elle est en constante augmentation, doublant tous les 10 ans, sans que l'on en connaisse avec précision les raisons $(1,2,3)$.

Le principal facteur prédisposant retrouvé dans cette étude est le terrain, notamment l'atopie. Le risque d'allergie est évalué de 20 à $40 \%$ si l'un des parents est allergique, 40 à $60 \%$ si les deux parents sont allergiques et 5 à $15 \%$ si aucun des parents n'est allergique (5).

La RA est une maladie inflammatoire chronique de la muqueuse de la fosse nasale, isolée, associée à d'autres manifestations (4). Elle se présente essentiellement sous deux formes, intermittente ou persistante $(1,2)$. Dans notre pratique, l'affection sévit sur un mode persistant (4). Le diagnostic repose sur la clinique (trépied classique : obstruction nasale, éternuements en salve, rhinorrhée aqueuse), l'interrogatoire étant primordial. Il est confirmé par des examens complémentaires biologiques ou par des tests cutanés.

Variations quantitatives du magnésium plasmatique et globulaire :

Il existe très peu d'observations sur le comportement du magnésium dans les RA. Les rares études rapportées soulignent la relation entre le déficit magnésique et certains cas d'allergie, telle l'allergie respiratoire, notamment la $\operatorname{RA}(6,7)$. 
Aussi, notre étude révèle que la magnésemie est abaissée dans la RA. L'hypomagnésemie concerne plus la fraction globulaire que plasmatique. Le sexe féminin paraît plus intéressé par cette hypomagnésemie.

Dans les rapports expérimentaux et cliniques entre magnésium et hypersensibilité, DURLACH (6) retrouve des cas d'hypomagnésemie et l'intérêt de la magnésothérapie dans la RA. Plusieurs études démontrent l'intérêt et l'efficacité du magnésium comme traitement dans la prise en charge des RA (8-11).

Le rôle du magnésium paraît bien établi dans le mécanisme de l'allergie. Le déficit expérimental du magnésium et l'allergie ont fait l'objet de plusieurs études ; notamment sur l'effet d'un régime pauvre en magnésium sur la toxicité de l'histamine chez le rat (12), sur le rôle du magnésium dans la libération d'histamine par les mastocytes (13) et le taux sérique d'anticorps réagines ( $\lg \mathrm{E})$ chez la souris en carence magnésique (14).

Il a été démontré que les réactions allergiques sont déclenchées par l'interaction, lors de sa réintroduction dans un organisme sensibilisé, entre des allergènes et des $\lg$ E spécifiques fixées sur des cellules par leurs récepteurs de forte affinité FceRl (mastocytes, basophiles, mais aussi éosinophiles, macrophages, cellules de Langherans...) et par leurs récepteurs de faible affinité FceRII (macrophages, éosinophiles, lymphocytes B...) (15).

Les cellules cibles de la réaction immédiate, anaphylactique, sont essentiellement les mastocytes portant les lg $E$ spécifiques de l'antigène. Leur activation entraîne leur dégranulation rapide avec libération dans les tissus de granulations contenant les médiateurs préformés (histamine, protéoglycanes, protéases neutres, cytokines). Dans un second temps sont synthétisés d'autres médiateurs (métabolites de l'acide arachidonique PGD2, LTC4/D4, (CysLT) et cytokines IL1, IL3-6, IL8, GM-CSF, TNFa).

Certains des médiateurs secrétés pendant la réaction immédiate sont chémotactiques sur les autres cellules de l'inflammation allergique (éosinophiles, lymphocytes, basophiles et macrophages) et stimulent l'expression par les cellules endothéliales de molécules d'adhésion. Les cellules recrutées vont libérer à leur tour leurs médiateurs cytotoxiques ou non (radicaux libres, ECP, EPO, CysLT, cytokines et chimiokines telles que IL-5, GM-CSF, éotaxine, RANTES, IL-8, IL-16...), aggravant les lésions tissulaires et pérennisant l'inflammation. Les lymphocytes qui infiltrent la muqueuse sont volontiers de type Th2, c'est-àdire sécrétant IL-4, IL-5, mais peu ou pas d'IL-2 et d'IFNg, à l'origine du recrutement, de l'activation et de l'augmentation de la survie (par inhibition de leur mort programmée ou aptoptose) de nombreux éosinophiles.

Ainsi, au regard de cette physiopathologie de l'allergie, le déficit en magnésium réduit l'information génétique et la formation des lymphocytes. II déprime la réponse immunitaire, exulte la réaction inflammatoire et la production des réagines ( $\lg E)$, tout en diminuant la phagocytose (6).

\section{CONCLUSION}

II se dégage de notre étude une relation certaine entre la magnésemie et la RA. Dans cette dernière, la magnésemie est abaissée et le taux globulaire est plus concerné. $\mathrm{Ce}$ déficit magnésique intervient de façon défavorable dans les différents stades de l'allergie.

La concordance clinico-biologique conforte l'idée du dosage de la magnésemie dans le bilan para clinique d'orientation et la prescription du magnésium comme traitement adjuvant dans la RA.

\section{REFERENCES}

1. Nathan RA, Maltzer EO, Derebery $\mathrm{J}$ and al. The prevalence of nasal symptoms attributed to allergies in the United States: findings from the burden of rhinitis in an American survery. Allergy Asthma Proc. 2008; 29: 600-8.

2. Weinmayr G, Forastice F, Weiland SK and al. International variation in prevalence of rhinitis and its relationship with sensitization to perennial and seasonal allergens. Eur Respir J. 2008; 32: 1250-61.

3. Bousquet J, Demoly P. Le traitement de la rhinite allergique persistante doit il être poursuivi au long cours ? Rev Off Soc Fr ORL. $2002 ; 74$ : 57-63.

4. Ondzotto G, Galiba J, Kouassi B, Ehouo F, Bamba M. Affections ORL associées à la rhinite allergique. Med Afr Noire. $2003 ; 50: 119-23$.

5. Koffi-Aka V, Ehouo F, Yotio A, Yapo G. Explorations des rhinites allergiques. Rev Odonto Stomatol Chir Maxillo Fac Afr. 1998 ; 5 : 42-6.

6 . Durlach J. Rapports expérimentaux et cliniques entre magnésium et hypersensibilité. Rev Fr Allergol Immunol Clin. 1975; 15 : 133-44.

7. Favennec F, Sonneville A, De Luca H, Castros A. Particular aspects of rhinitis and rhino-conjunctivis. Eur Ann Allergy Clin Immunol. 2003; 35: 99-102.

8. Gaby AR. Intravenous nutrient therapy: the "Myers cocktail". Altern Med Rev. 2002; 7: 389-403.
9. Miyake $\mathrm{Y}$, Sasaki S, Ohya $\mathrm{Y}$ and al. Dietary intake of seaweed and minerals and prevalence of allergic rhinitis in Japanese pregnant females: baseline data from the Osaka Maternal and Child Health Study. Ann Epidemiol 2006; 16: 614 21. 10. Miadonna A, Tedeschi A, Parravicini P, Lorini M. Evaluation of the efficacity of the magnesium salt of NAAGA the treatment of acute rhinitis. Clin Ter. 1989; 131: 173-6.

11. Cippola C, Occhionero T, Orciari P, Lugo G, D’Antuono G. Magnesium pidolate in the treatment of seasonal allergic rhinitis. Preliminary data. Magnes Res. 1990; 3: 109-12.

12. Geelen MJH, Van Logten MJ. Effet d'un régime pauvre en magnésium sur la toxicité de l'histamine chez le rat. Rev Fr Allergol Immunol Clin. 1975; 15: 155-7.

13. Marcelli $D$, Renoux ML. Rôle du magnésium dans la libération d'histamine par les mastocytes. Rev Fr Allergol Immunol Clin. $1975 ; 15: 151-4$

14. Prouvost Danon A, Larvor $P$. Taux sérique d'anticorps réagines $(\mathrm{lgE})$ chez la souris en carence magnésique. Rev Fr Allergol Immunol Clin. 1975; 15: 147-50. 15. Bousquet J, Vignola AM, Campbell AM, Michel FB. Pathophysiology of allergic rhinitis. Int Arch Allergy Immunol. 1996; 110-218. 